\title{
Detecting the Contours via a New Approximation of the Gradient
}

\author{
Mohamed Lagzouli \\ Equip of Cryptography and \\ Information \\ Processing, Department of \\ Mathematics \\ Faculty of Sciences, Ibn Tofail \\ University, \\ Kenitra, Morocco
}

\author{
Mustapha Rachidi \\ Equip of DEFA - Department of \\ Mathematics and Informatics, \\ Faculty of Sciences \\ Moulay Ismail University, \\ Meknes, Morocco
}

\author{
Youssfi Elkettani \\ Equip of Cryptography and \\ Information \\ Processing, Department of \\ Mathematics \\ Faculty of Sciences, Ibn Tofail \\ University \\ Kenitra, Morocco
}

\begin{abstract}
Contours detection is a key component of many image processing and computer vision. This paper proposes and validates a new efficient gradient method for detecting the contours in grayscale image. This method is based on the average of two derivatives, obtained from two different steps. This mathematical formulation, derived from a discrete numerical differentiation of image, plays a central role in this method. There are presented some operators and mask of discrete functions, which are effective for the detection of contours. Comparison of the mask obtained using the two derivatives operator, with the usual linear masks, allows us to show the efficiency of the new mask.
\end{abstract}

\section{General Terms}

Derivative operator, Finite difference, Gradient, Grayscale image, Taylor series,

\section{Keywords}

Steps, Mask, Operator, Mixture of two steps, Convolution kernel, Discrete functions

\section{INTRODUCTION}

The gradient edge detection by filtering, applied on discrete functions, is based on the determination of an extremum of the first derivative (or the second derivative). This method has been studied by various authors (see for example [1,2,3] and references therein). Thereby, Modestino and Fries proposed a Wiener filter to detect the contours (see [4]). The purpose of this filtering is to perform the non-noisy image, using the Laplacian operation. This technic is followed by the zeros detection combined with thresholding. On the other hand, the derivation obtained by finite difference of an image or discrete functions is discrete in nature. The preceding approach have been used, particularly by Bohlen et al., T. Kapur (see $[5,6,7,8,9]$ ), to approximate the derivatives by divided difference. These derivatives are calculated by convolution of the image with a mask.

Since the appearance of promising results using the gradient of digital images, many authors have investigated this topic.

Especially some interesting filters has been proposed by Sobel, Prewitt, Kirch, Robinson, Shen-Castan, these filters are studied in $[10,11,12,13,14]$. The methods mentioned above are the most currently used in the detection of contours, with the aid of masks. However, these methods suffer from lack of the mathematical definition of masks. For example, some rotation in the mask has been introduced by Prewitt, Kirch and Sobel for determining these masks. Therefore, as shown in the above discussion, the mathematical methods play a central role in the detection problem of the contours.

The main concerns of this paper is to propose a new method of gradient called derivative operator, which can be applied to every discrete function, signal and matrix, in the aim to calculate the gradient. This derivative operator permits to find a new powerful mask, able to detect the contours of grayscales images.

The content of this paper is organized as follows: It starts by presenting the Lagrange interpolation of a discrete function. Some derivative operators of these functions are presented. This paper introduces a new method of gradient with mixture of two steps. The comparison of the proposed operator with other operators permits to show the efficiency of the new method.

\section{METHODS AND BASIC MATERIALS 2.1 The Method of Discrete Functions $d_{2 n}$}

Let $\mathrm{P}$ [resp. $\mathrm{P}_{2 n}$ ] denotes the vector space of restrictions of polynomials functions on $\square$ [resp. of degree at most 2n] defined on $\square$ (see [15]). For every $\varphi \in \mathrm{P}$, let $\varphi(m)=\sum_{i=1, n} a_{i} m^{i}$ for $m \in \square$ and for every $\mathrm{z}$ in $\square$ $\tilde{\varphi}(z)=\sum_{i=1, n} a_{i} z^{i}$. Let $\alpha \in \square$ and consider the application $\mathrm{T}^{\alpha}: \mathrm{P} \rightarrow \mathrm{P}$ defined by

$$
\left(\mathrm{T}^{\alpha} \varphi\right)(m)=\tilde{\varphi}(m-\alpha)
$$

For every $m \in \square$. It is well known that $\mathrm{T}^{\alpha}$ is an interpolator. The application $\partial: \mathrm{P} \rightarrow \mathrm{P}$ defined by $(\partial \varphi)(m)=\tilde{\varphi}^{\prime}(m)$, for every $m \in \square$, is called derivative operator. Since $\varphi$ is a polynomial, then according to (1) it derives easily that $\mathrm{T}^{\alpha} \varphi$ is also a polynomial.

Proposition. The derivative operator is fitted with a convolution of simple convergence $\partial \in L_{*}(\mathrm{P})$. More precisely, the set $P$ is a real vector space, stable by translation actions. Moreover, the two operators $\partial$ and $\mathrm{T}^{\alpha}$ are linear. If $\mathrm{T}^{n}(n \in \square)$ is a translation and $\varphi \in \mathrm{P}$ then, $\mathrm{T}^{n} * \varphi$ is a polynomial of $P$.

The preceding Proposition permits to show the following equality,

$$
\partial\left(\mathrm{T}^{n} * \varphi\right)(m)=\tilde{\varphi}^{\prime}(m-n)=\left(\mathrm{T}^{n} * \partial \varphi\right)(m) .
$$


Thus, the operator $\partial$ commutes with translations $\mathrm{T}^{n} \quad(n \in \square$ ) .

\subsection{The Derivation in the Sense of Convolution}

For every $\varphi \in \mathrm{P}_{2 n}(\square)$, consider Lagrangian interpolation polynomial of degree $2 \mathrm{n}$, in $2 \mathrm{n}+1$ arbitrary points of $\square$. Given $m_{0} \in \square$, then $\varphi$ can be evaluated at $m_{0}+p$ ( $0 \leq|p| \leq n)$, as follows. For every $x \in \square$, let

$$
\tilde{\varphi}(x)=\sum_{0 \leq|p| \leq n} \varphi\left(m_{0}+p\right)\left[\prod_{k \neq p} \frac{x-\left(m_{0}+k\right)}{p-k}\right]
$$

Therefore, the derivative of $\tilde{\varphi}$ at $x \in \square$, is given by,

$$
\tilde{\varphi}^{\prime}(x)=\sum_{0 \leq p \mid \leq n} \varphi\left(m_{0}+p\right) \frac{1}{\prod_{k \neq p}(p-k)}\left[\sum_{i \neq p}\left(\prod_{k \neq i, p}\left(x-\left(m_{0}+k\right)\right)\right)\right]
$$

And for $x=m_{0}$ the derivative is given by,

$$
\tilde{\varphi}^{\prime}\left(m_{0}\right)=\sum_{0 \leq \leq p \mid \leq n} \varphi\left(m_{0}+p\right) \frac{1}{\prod_{k \neq p}(p-k)}\left[\sum_{i \neq p}\left(\prod_{k \neq i, p}(-k)\right)\right]
$$

The different products involved in the preceding expression

(2) can be evaluated explicitly. Indeed, since

$$
\prod_{k \neq i, p}(-k)= \begin{cases}0 & \text { if } p \neq 0 \text { and } i \neq 0 \\ \frac{(-1)^{n}}{-i}(n !)^{2} & \text { if } p=0 \text { and } i \neq 0 \\ (-1)^{n}(n !)^{2} & \text { if } p=0 \text { and } i=0\end{cases}
$$

then $\prod_{k \neq p}(p-k)=(-1)^{n-p}(n+p) !(n-p)$ !. On the other hand, the term of the sum corresponding to $p=0$ is equal 0 , thus the substitution of the preceding products in (2), gives

$$
\varphi^{\prime}\left(m_{0}\right)=\sum_{0 \leq 1 p \mid \leq n} \varphi\left(m_{0}+p\right) \frac{(-1)^{p}}{-p} \frac{(n !)^{2}}{(n+p) !(n-p) !}
$$

The index change $p^{\prime}=-p$ allows to get

$$
\varphi^{\prime}\left(m_{0}\right)=\sum_{0 \leq 1 p \mid \leq n} \varphi\left(m_{0}-p\right) d_{2 n}(p)=\sum_{0 \leq 1 p \mid \leq n} \varphi(p) d_{2 n}\left(m_{0}-p\right)
$$

where

$$
d_{2 n}(p)= \begin{cases}0 & \text { if } \mathrm{p}=0 \text { or }|p|>\mathrm{n} \\ \frac{(-1)^{p}}{p} \frac{(n !)^{2}}{(n+p) !(n-p) !} & \text { if } 0<|p| \leq n\end{cases}
$$

Then the discrete derivative (3)-(4) can be given by the following formula $\varphi^{\prime}=\varphi^{*} d_{2 n}$, where $*$ is the discrete convolution product.
Expression (5) shows that each operator $d_{2 n}$ can be identified with the $2 n+1$ values obtained from (5). The first operators $d_{2 n}$ are given by,

$$
\begin{array}{lll}
d_{2}=\left(\frac{1}{2}, 0,-\frac{1}{2}\right), & \text { for } & \mathrm{n}=1 \\
d_{4}=\left(-\frac{1}{12}, \frac{2}{3}, 0,-\frac{2}{3}, \frac{1}{12}\right), & \text { for } & \mathrm{n}=2 \\
d_{6}=\left(\frac{1}{60},-\frac{1}{10}, \frac{1}{4}, 0,-\frac{1}{4}, \frac{1}{10},-\frac{1}{60}\right), & \text { for } & \mathrm{n}=3 \\
d_{8}=\left(-\frac{1}{280}, \frac{4}{105},-\frac{1}{5}, \frac{4}{5}, 0,-\frac{4}{5}, \frac{1}{5},-\frac{4}{105}, \frac{1}{280}\right), \text { for } \mathrm{n}=4
\end{array}
$$

Hence, the applications $d_{2 n}$ can be viewed as a derivative operators of a discrete function on $\mathbf{Z}$. Since the set of these operators growth with $\mathrm{n}$, let focus in the sequel on the first of them.

Recall that the local convolution operation is a central key in modern image processing. That is, the basic idea is that a window of some finite size and shape -the support - can be scanned across the image. The output pixel value is the weighted sum of the input pixels within the window, where the weights are the values of the filter assigned to every pixel of the window itself. The window with its weights is called the convolution kernel. If the filter $h[j, k]$ is zero outside the (odd sized rectangular) window of size $J \times K$ centered at the origin $\left\{j=-J_{0},-J_{0+1}, \ldots,-1,0,1, \ldots, J_{0-1}, J_{0} ; k=-K_{0},-K_{0+1}, \ldots,-\right.$ $\left.1,0,1, \ldots, K_{0-1}, K_{0}\right\}$, then the convolution can be written under the following form

$$
s[m, n]=f[m, n] * h[m, n]=\sum_{j=-J_{0}}^{J_{0}} \sum_{k=-k_{\circ}}^{k_{\circ}} h[j, k] f[m-j, n-k]
$$

where $f$ is the input image and $s$ is the output image. The new idea is to propose below a new technique based on the concept of step and convolution kernel, for studying the digital image.

\subsection{Derivation by Mixture of Two Steps}

Let first recall some basic and useful definitions. Let $f$ be a function defined on an open set $\Omega$. It is well known that the gradient $\nabla f(x)$ of $f$ at $x$, is the vector pointing in the direction of maximum growth of the function $f$, whose modulus is given precisely by the maximum growth rate of $f$ at $x$. In the case of Cartesian coordinates, the gradient is given by,

$$
\nabla f(x, y)=\left(\frac{\partial f}{\partial x}, \frac{\partial f}{\partial y}\right)
$$

The next goal is to evaluate $f$ at the mesh node $x_{i}$, by setting $f_{i}=f\left(x_{i}\right)$.

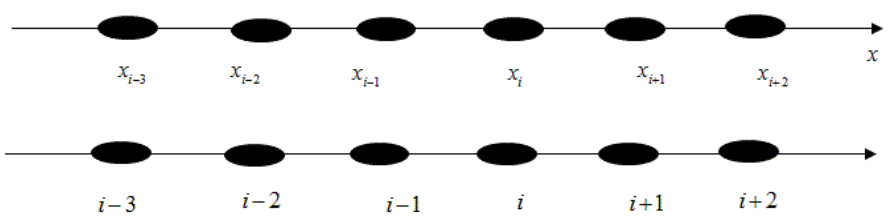

Figure 1: Mesh points by the Taylor series 
To this aim let consider a derivative operator $I_{M 2 P}: \square^{5} \rightarrow \square$ representing a new approximation of gradient, defined by $I_{M 2 P}(\vec{f}) \square \overrightarrow{\operatorname{Grad}}(f)=\nabla f$ where $\vec{f}$ is the vector column $\vec{f}=\left(f_{i-2}, f_{i-1}, f_{i}, f_{i+1}, f_{i+2}\right)^{t}$.

The idea is to use the notion of finite differences to calculate the derivative of a function or matrix image. Indeed, the results of $[16,17]$ permit to show how to exploit this technic in digital images. Then, with the aid of the average of a mixture of two different steps to explicit the operator $I_{M 2 P}$.

More precisely, let apply this notion of step for deriving the new gradient operator. The main purpose is to propose an adequate gradient approximation, using the Taylor series of the function $f$.

Theorem (Approximation of the gradient with a mixture of two steps) The gradient of a discrete function $f$ (matrix or polynomial) at $x$, is obtained by applying the operator $I_{M 2 P}$, where $I_{M 2 P}=\frac{1}{8}\left(\begin{array}{lllll}-1 & -2 & 0 & 2 & 1\end{array}\right)^{t}$ is the operator obtain by using a mixture of two steps.

Proof: The Taylor series of $f$ at point $\mathrm{x}_{\mathrm{i}}$ (Figure 1) gives the

following two expressions,

$f_{i+1}=f_{i}+\left(x_{i+1}-x_{i}\right) \frac{\partial f}{\partial x}\left(x_{i}\right)+\frac{\left(x_{i+1}-x_{i}\right)^{2}}{2} \frac{\partial^{2} f}{\partial x^{2}}\left(x_{i}\right)+o\left(\left(x_{i+1}-x_{i}\right)^{2}\right)(9)$

$f_{i-1}=f_{i}+\left(x_{i-1}-x_{i}\right) \frac{\partial f}{\partial x}\left(x_{i}\right)+\frac{\left(x_{i-1}-x_{i}\right)^{2}}{2} \frac{\partial^{2} f}{\partial x^{2}}\left(x_{i}\right)+o\left(\left(x_{i-1}-x_{i}\right)^{2}\right)^{(10)}$

In fact, these expressions give the derivative of $f$ at node $i$. If the step $\Delta x=\left(x_{i+1}-x_{i}\right)=-\left(x_{i-1}-x_{i}\right)=1$, is considered in equations (9) - (10) we obtain,

$$
\begin{aligned}
& f_{i+1}-f_{i-1}=\left[f_{i}+\Delta x \frac{\partial f}{\partial x}\left(x_{i}\right)+\frac{\Delta x^{2}}{2} \frac{\partial^{2} f}{\partial x^{2}}\left(x_{i}\right)+o\left(\Delta x^{2}\right)\right]- \\
& {\left[f_{i}-\Delta x \frac{\partial f}{\partial x}\left(x_{i}\right)+\frac{\Delta x^{2}}{2} \frac{\partial^{2} f}{\partial x^{2}}\left(x_{i}\right)+o\left(\Delta x^{2}\right)\right]=2 \Delta x \frac{\partial f}{\partial x}\left(x_{i}\right)}
\end{aligned}
$$

Therefore, $\frac{\partial f}{\partial x}\left(x_{i}\right)=\frac{f_{i+1}-f_{i-1}}{2 \Delta x}$, where

$\Delta x=\left(x_{i+1}-x_{i}\right)=-\left(x_{i-1}-x_{i}\right)=1$, which means that,

$$
\overrightarrow{\operatorname{Grad}}_{1}\left(f\left(x_{i}\right)\right)=\frac{f_{i+1}-f_{i-1}}{2}
$$

Now, in the step let $\Delta x=\left(x_{i+2}-x_{i}\right)=2$, which verifies also $\Delta x=-\left(x_{i-2}-x_{i}\right)=2$. Hence, the Taylor series provide the equations,

$$
\begin{gathered}
f_{i+2}=f_{i}+\left(x_{i+2}-x_{i}\right) \frac{\partial f}{\partial x}\left(x_{i}\right)+\frac{\left(x_{i+2}-x_{i}\right)^{2}}{2} \frac{\partial^{2} f}{\partial x^{2}}\left(x_{i}\right)+o\left(\left(x_{i+2}-x_{i}\right)^{2}\right) \\
f_{i-2}=f_{i}+\left(x_{i-2}-x_{i}\right) \frac{\partial f}{\partial x}\left(x_{i}\right)+\frac{\left(x_{i-2}-x_{i}\right)^{2}}{2} \frac{\partial^{2} f}{\partial x^{2}}\left(x_{i}\right)+o\left(\left(x_{i-2}-x_{i}\right)^{2}\right)
\end{gathered}
$$

From (12) and (13) the difference gives,

$$
\begin{aligned}
& f_{i+2}-f_{i-2}=\left[f_{i}+\Delta x^{\prime} \frac{\partial f}{\partial x}\left(x_{i}\right)+\frac{\Delta x^{\prime 2}}{2} \frac{\partial^{2} f}{\partial x^{2}}\left(x_{i}\right)+o\left(\Delta x^{\prime 2}\right)\right]- \\
& {\left[f_{i}-\Delta x^{\prime} \frac{\partial f}{\partial x}\left(x_{i}\right)+\frac{\Delta x^{\prime 2}}{2} \frac{\partial^{2} f}{\partial x^{2}}\left(x_{i}\right)+o\left(\Delta x^{\prime 2}\right)\right]=2 \Delta x^{\prime} \frac{\partial f}{\partial x}\left(x_{i}\right)}
\end{aligned}
$$

Therefore, $\frac{\partial f}{\partial x}\left(x_{i}\right)=\frac{f_{i+2}-f_{i-2}}{2 \Delta x^{\prime}}$, where

$\Delta x^{\prime}=\left(x_{i+2}-x_{i}\right)=-\left(x_{i-2}-x_{i}\right)=2$. Hence,

$$
\overrightarrow{\operatorname{Grad}}_{2}\left(f\left(x_{i}\right)\right)=\frac{f_{i+2}-f_{i-2}}{4}
$$

Equation (11) and (14) allow us to deduce the following expression,

$$
\begin{aligned}
\frac{1}{2}\left(\overrightarrow{\operatorname{Grad}}_{1}\left(f\left(x_{i}\right)\right)+\right. & \left.\overrightarrow{\operatorname{Grad}_{2}}\left(f\left(x_{i}\right)\right)\right) \\
& =\frac{1}{2}\left(\frac{f_{i+1}-f_{i-1}}{2}+\frac{f_{i+2}-f_{i-2}}{4}\right) \\
& =\frac{1}{8}\left[f_{i+2}+2 f_{i+1}-2 f_{i-1}-f_{i-2}\right]=I_{M 2 p}(\vec{f})
\end{aligned}
$$

Here $I_{M 2 P}=\frac{1}{8}\left(\begin{array}{lllll}-1 & -2 & 0 & 2 & 1\end{array}\right)^{t}$ is the new operator obtained from a mixture of two steps. End of the proof.

Proposition. Let $f$ be a function defined on an open set $\Omega$.If the nodes are uniformly spaced the gradient of $f$ is given by,

$$
I_{M 2 P}(f)=\overrightarrow{\operatorname{Grad}}(f)=\frac{1}{2}\left(\overrightarrow{\operatorname{Grad}}_{1}(f)+\overrightarrow{\operatorname{Grad}}_{2}(f)\right),
$$

where, $\overrightarrow{\operatorname{Grad}}_{1}(f)$ is the gradient of the function $f$ with the step equal to 1 and $\overrightarrow{G r a d}_{2}(f)$ is the gradient of the function $f$ with the step equal to 2 .

\section{THE PROPOSED MASK}

This section show that a new derivative operator $I_{M 2 P}$, admits the same properties as $d_{2 n}$ and it is also able to detect contours in digital images (see $[18,19,20,21,22])$. The proposed mask based on the technique of calculating the average gradient $I_{M 2 P}$. This mask - noted $M I_{M 2 P}$ - is constructed by the convolution between a smoothing operator and the operator $I_{M 2 P}$.

Let $h_{x}$ denotes a horizontal derivative filter, $h_{y}$ denotes a vertical derivative filter, and $h_{\theta}$ denotes the arbitrary angle derivative filter, then the computation of the directional derivatives of $f$ is done by the convolution of $f$ with the kernel of $h_{x}$ or $h_{y}$ as follows, $\frac{\partial f}{\partial x}(x, y) \square\left(f * h_{x}\right)(x, y)$ and $\frac{\partial f}{\partial y}(x, y) \square\left(f * h_{y}\right)(x, y)$, where 
$h_{x}=I_{M 2 P}\left(\begin{array}{lllll}1 & 1 & 1 & 1 & 1\end{array}\right)=\frac{1}{8}\left(\begin{array}{ccccc}-1 & -1 & -1 & -1 & -1 \\ -2 & -2 & -2 & -2 & -2 \\ 0 & 0 & 0 & 0 & 0 \\ 2 & 2 & 2 & 2 & 2 \\ 1 & 1 & 1 & 1 & 1\end{array}\right)$

Let $h_{y}=h_{x}^{t}$ denotes the transpose of $h_{x}$,

$$
h_{y}=\frac{1}{8}\left(\begin{array}{ccccc}
1 & 2 & 0 & -2 & -1 \\
1 & 2 & 0 & -2 & -1 \\
1 & 2 & 0 & 0 & -1 \\
1 & 2 & 0 & -2 & -1 \\
1 & 2 & 0 & -2 & -1
\end{array}\right)=h_{x}^{t}
$$

Therefore, the equalities $f_{x}=f * h_{x}$ and $f_{y}=f * h_{y}$, evaluated at $x_{i}$, represent an approximation of the partial derivative of the function $f$. In other works, the real numbers $f_{x}=f_{x}\left(x_{i}\right)=f\left(x_{i}\right) * h_{x}$ and $f_{y}=f_{y}\left(x_{i}\right)=f\left(x_{i}\right) * h_{y}$ approximate the partial derivative of the function $f$. The discrete gradient filters and the gradient, $\nabla f(x, y)$, generate the same effect on a grayscale image. Moreover, the gradient is given by,

$$
\nabla f(x, y)=\frac{\partial f}{\partial x} \overrightarrow{i_{x}}+\frac{\partial f}{\partial y} \overrightarrow{i_{y}}=\left(h_{x} * f\right) \overrightarrow{i_{x}}+\left(h_{y} * f\right) \overrightarrow{i_{y}}
$$

where $\overrightarrow{i_{x}}$ and $\overrightarrow{i_{y}}$ are unit vectors in the horizontal and vertical direction, respectively.

This leads to the descriptions of the gradient magnitude and the gradient direction given by $|\nabla f|=\sqrt{\left(h_{x} * f\right)^{2}+\left(h_{y} * f\right)^{2}}$ and $f_{\theta}=\arctan \left\{\left(h_{x} * f\right) /\left(h_{y} * f\right)\right\}$. A new mask $M I_{M 2 P}$ more efficient for detecting the contours is obtained by applying successively the operator $I_{M 2 P}$ and the smooth operator $\left(\begin{array}{lllll}1 & 1 & 1 & 1 & 1\end{array}\right)^{t}$. utilized for detecting the contours in the grayscale image (Figure 6).

The derivative operator in formula (7) is applied to the original signal. The result presents in the following Figure 2,

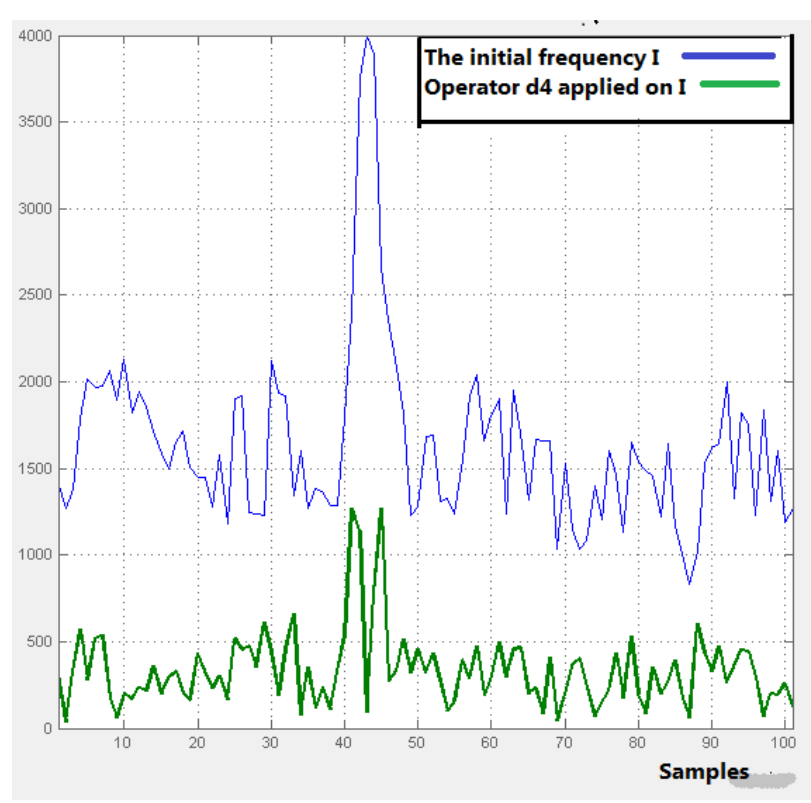

Figure 2: The initial frequency $I$ present in blue and green present operator $d_{4}$ applied in $I$.

\begin{tabular}{|c|c|c|c|c|c|c|c|c|c|c|c|c|c|c|c|c|c|c|c|c|c|}
\hline & \multicolumn{3}{|r|}{$M d_{2}$} & \multicolumn{5}{|c|}{$M I_{M 2 P}$} & \multicolumn{5}{|c|}{$M d_{4}$} & \multicolumn{8}{|c|}{$M d_{6}$} \\
\hline Masks & $\frac{1}{2}$ & $\left(\begin{array}{c}-1 \\
0 \\
1\end{array}\right.$ & $\left.\begin{array}{cc}-1 & -1 \\
0 & 0 \\
1 & 1\end{array}\right)$ & $\frac{1}{8}\left(\begin{array}{c}-1 \\
-2 \\
0 \\
2 \\
1\end{array}\right.$ & $\begin{array}{c}-1 \\
-2 \\
0 \\
2 \\
1\end{array}$ & $\begin{array}{c}-1 \\
-2 \\
0 \\
2 \\
1\end{array}$ & $\begin{array}{c}-1 \\
-2 \\
0\end{array}$ & $\left.\begin{array}{c}-1 \\
-2 \\
0 \\
2 \\
1\end{array}\right)$ & $\left(\begin{array}{c}-\frac{1}{12} \\
\frac{2}{3} \\
0 \\
-\frac{2}{3} \\
\frac{1}{12}\end{array}\right.$ & $\begin{array}{l}-\frac{1}{12} \\
\frac{2}{3} \\
0 \\
-\frac{2}{3} \\
\frac{1}{12}\end{array}$ & $\begin{array}{c}-\frac{1}{12} \\
\frac{2}{3} \\
0 \\
-\frac{2}{3} \\
\frac{1}{12}\end{array}$ & $\begin{array}{c}-\frac{1}{12} \\
\frac{2}{3} \\
0 \\
-\frac{2}{3} \\
\frac{1}{12}\end{array}$ & $\left.\begin{array}{c}-\frac{1}{12} \\
\frac{2}{3} \\
0 \\
-\frac{2}{3} \\
\frac{1}{12}\end{array}\right)$ & $\left(\begin{array}{c}\frac{1}{60} \\
\frac{-1}{10} \\
\frac{1}{4} \\
0 \\
\frac{-1}{4} \\
\frac{1}{10} \\
\frac{-1}{60}\end{array}\right.$ & $\begin{array}{c}\frac{1}{60} \\
\frac{-1}{10} \\
\frac{1}{4} \\
0 \\
\frac{-1}{4} \\
\frac{1}{10} \\
\frac{-1}{60}\end{array}$ & $\begin{array}{c}\frac{1}{60} \\
\frac{-1}{10} \\
\frac{1}{4} \\
0 \\
\frac{-1}{4} \\
\frac{1}{10} \\
\frac{-1}{60}\end{array}$ & $\begin{array}{c}\frac{1}{60} \\
\frac{-1}{10} \\
\frac{1}{4} \\
0 \\
\frac{-1}{4} \\
\frac{1}{10} \\
\frac{-1}{60}\end{array}$ & $\begin{array}{c}\frac{1}{60} \\
\frac{-1}{10} \\
\frac{1}{4} \\
0 \\
\frac{-1}{4} \\
\frac{1}{10} \\
\frac{-1}{60}\end{array}$ & $\begin{array}{l}\frac{1}{60} \\
\frac{-}{10} \\
\frac{1}{4} \\
0 \\
\frac{1}{4} \\
\frac{1}{10} \\
\frac{-}{60}\end{array}$ & $\begin{array}{l}\frac{1}{50} \\
\frac{-1}{0} \\
\frac{1}{4} \\
0 \\
\frac{-1}{4} \\
\frac{1}{0} \\
\frac{-1}{50}\end{array}$ & $\begin{array}{c}\frac{1}{60} \\
\frac{-1}{10} \\
\frac{1}{4} \\
0 \\
\frac{-1}{4} \\
\frac{1}{10} \\
\frac{-1}{60}\end{array}$ \\
\hline
\end{tabular}

Table 1 : Different types of masks.

\section{RESULTS}

The results obtained have important efficiency. First of all, the formulas (6), (7), (8) and (15) are used for computing the gradient of the signal. These formulas called also the derivatives operators, are used to detect the peaks in signal. In the sequel the different masks are obtained by using the operators $d_{2}, d_{4}, d_{6}$ and $I_{M 2 P}$. These masks ( see Table 1) are
The derivative operator in formula (8) is applied to the original signal. The result presents in the following Figure 3, 


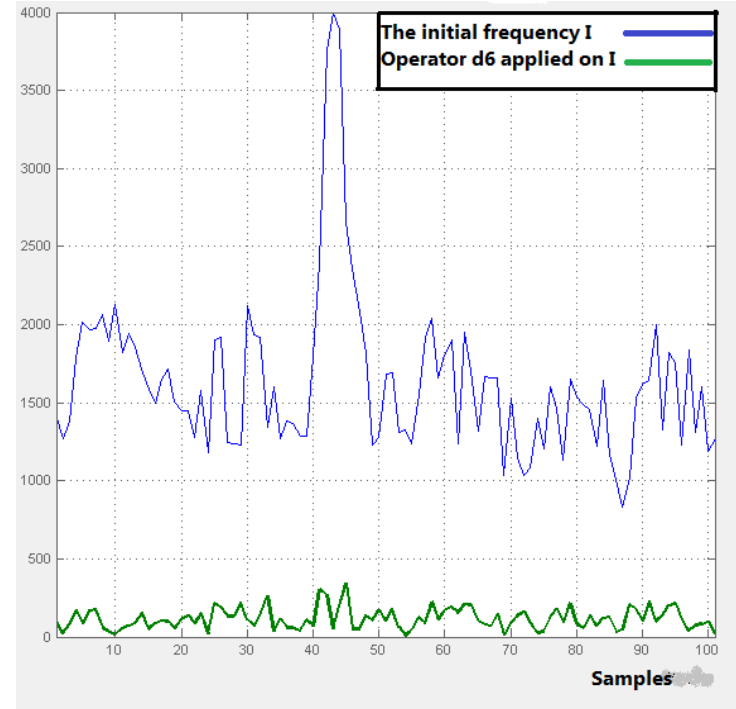

Figure 3: The initial frequency $I$ present in blue and green present operator $d_{6}$ applied in $I$.

Now, the derivative operator in Formula (15) is applied to the original signal. The result presents in the following Figure 4,

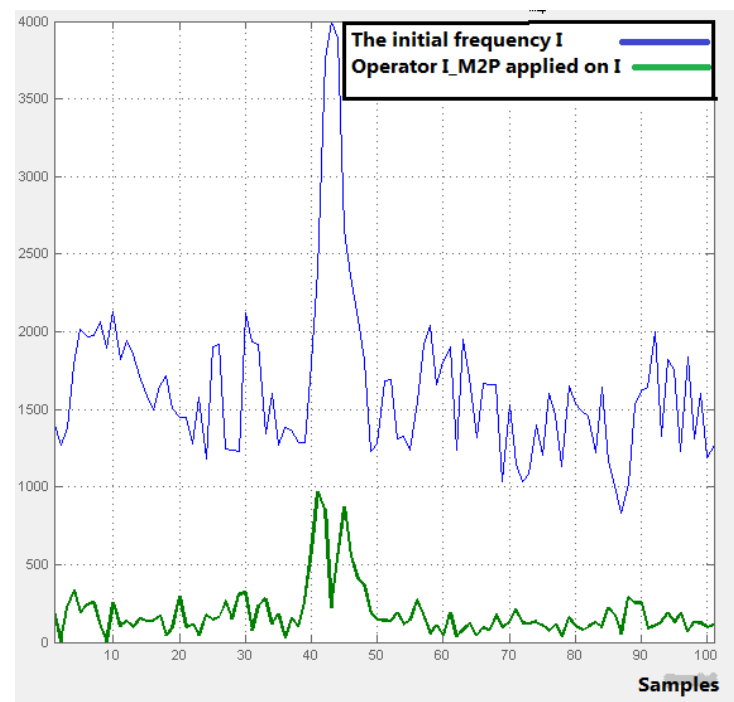

Figure 4: The initial frequency $I$ present in blue and green present operator $I_{M 2 P}$ applied in $I$.

Figure 5 compare the operators $d_{4}, d_{6}$ and $I_{M 2 P}$, when they are applied to the original signal.

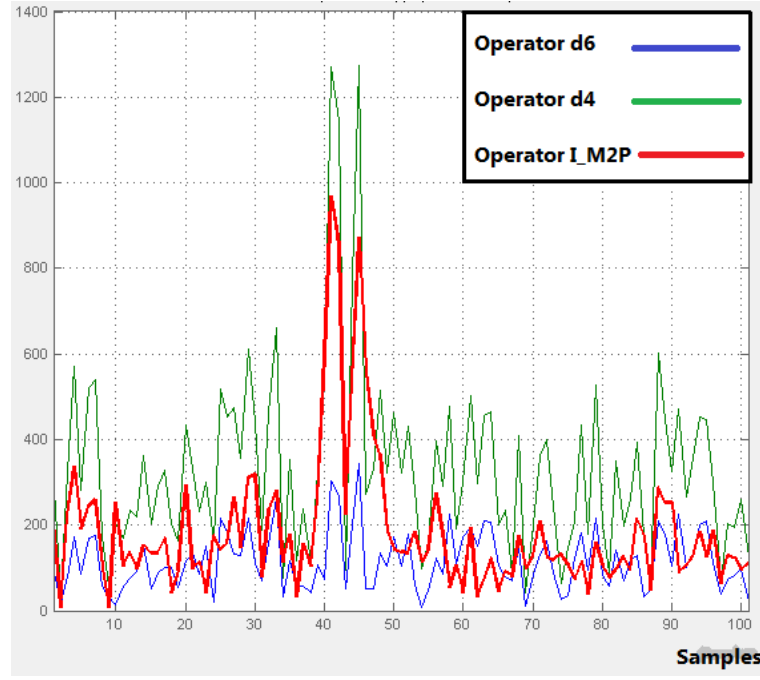

Figure 5: The comparison of the 3 operators $d_{4}, d_{6}$ and $I_{M 2 P}$ applied in original signal.

The masks $M d_{2}, M d_{4}$ and $M d_{6}$ are built respectively from the formulas (6), (7) and (8). The filter $M d_{2}$ obtained by (6) is identical to the filter of Prewitt and the proposed mask $M I_{M 2 P}$ is constructed with the aid of formula (15).

The various masks of Table 1, obtained using the preceding formulas are applied to detect the contours in grayscale image as shown and described in Figure 6.

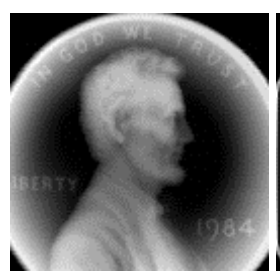

(i)

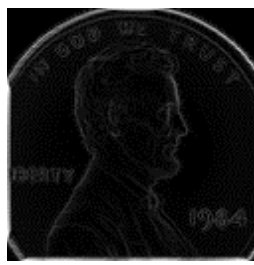

(iv)

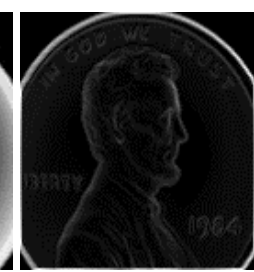

(ii)

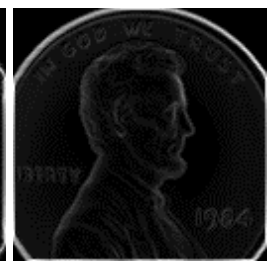

(v)

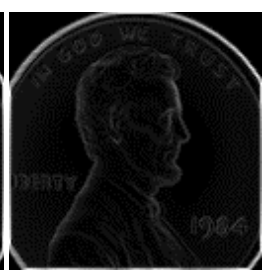

(iii)

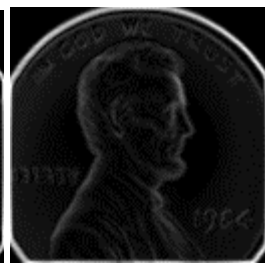

$(v i)$
Figure 6: (i) shows the original image, (ii) the image is filtered by $M d_{2}$ (Prewitt filter), (iii) the image is filtered by $M d_{4}$, (iv) the image is filtered by $M d_{6}$, (v) the image is filtered by Sobel mask and (vi) the same image is filtered

$$
\text { by } M I_{M 2 P} \text {. }
$$

\section{DISCUSSION AND CONCLUSION}

Three operators $d_{4}, d_{6}$ and $I_{M 2 P}$ are used to deduce the peaks in Figure 2, Figure 3 and Figure 4. The masks in Table1 are utilized for deriving the contours in the grayscale image (see Figure 5). In Figure 4 the peak becomes negligible after applying the operator $d_{6}$. This shows that the derivative 
operators $d_{2 n}$ are not valid for all $\mathrm{n}$. For $n \geq 3$, the filter built by the operator $d_{2 n}$ becomes a low derivative operator.

Figure 2 and Figure 4 show that the operator $d_{4}$ and $I_{M 2 P}$ (green color) are able to detect the high transitions in the original signal (blue color), and the operator $d_{6}$ in Figure 3 is unable to detect them.

A conclusion also seen is that the proposed operator $I_{M 2 P}$ is better than $d_{4}$ because the later detects small transitions and considers them as peaks. But this is not true since the original signal has one peak. However, the operator $I_{M 2 P}$ is more realistic. Indeed, Figure 6 highlights the mask $M I_{M 2 P}$ compared to other masks. The results of this comparison are favorable to the proposed mask to detect the contours.

Figure 5 and Figure 6 show that the operators $d_{4}$ and $I_{M 2 P}$ are able to detect contours in two-dimensional case and peaks in mono-dimensional. Otherwise, for $d_{2 n}$, with $n \geq 3$, it is not possible to detect it efficiently. However, Figure 6 highlights the mask $M I_{M 2 P}$ compared to the masks $M d_{2}$ (the same of Prewitt filter), $M d_{4}$ and $M d_{6}$. The results in Figure 6 show that the mask $M I_{M 2 P}$ is better than $M d_{2 n}$. Figure 6 allows also to compare this mask with some known masks in the literature, for example Sobel's mask and Prewitt's mask. The results of this comparison show that the proposed mask, for detecting the contours, is more efficient.

\section{REFERENCES}

[1] Jean-Paul Chehab. 2009. Analyse discrète : Interpolation Polynomiale, 3-6.

[2] Simai He, Zhening LI and Shuzhong Zhang. 2010. Approximation Algorithms for Discrete Polynomial Optimization.

[3] V. Torre and T. A. Poggio. On Edge Detection. 1986. In IEEE Transactions on Pattern Analysis and Machine intelligence, PAMI-8, 2(March 1986), 147- 163.

[4] J .W. Modestino, R .W. Fries . 1977. Edge detection in noisy images using recursive digital filter. Compt. Graphics and Image Processing, 6(1977), 409- 433.

[5] T. Bohlen and Saenger E H. 2006. Accuracy of heterogeneous staggered-grid finite- difference modeling of Rayleigh waves. Geophysics 71(2006), T109-T115.

[6] T. Kapur, L. Yezzi, and L. Zöllei. 2001. A variational framework for joint segmentation and registration, in the IEEE Workshop on Mathematical Methods in Biomedical Image Analysis (MMBIA 2001), IEEE Computer Society (2001), 44-51.

[7] Yang Liu and Mrinal K. Sen. 2009. Numerical modeling of wave equation by a truncated high-order finitedifference method, Earthq Sci 22(2009), 205-213.

[8] Okunuga, S. A., and Akanbi M, A. 2004. Computational Mathematics. First Course, WIM Pub. Lagos, Nigeria.

[9] Jae H. Park. 1999. Chebyshev Approximation of Discrete Polynomials and Splines. Blacksburg, Virginia.18-23
[10] A. Beghdadi and A. Le Negrate. 1989. Contrast Enhancement Technique Based on Local Detection of Edges. Computer Vision, Graphics, and Images Processing. 46(1989), 162-174.

[11] M. Lagzouli, Y. Elkettani. 2012. La Détection des Contours dans les Images Numériques. au colloque 7 ème Rencontre Nationale des jeunes chercheurs en Physique « RNJCP7 » à Faculté des sciences Ben M'sik Casablanca -Maroc.

[12] N. Paragios and R. Deriche. 2000. Geodesic active contours and level sets for the detection and tracking of moving objects, IEEE Trans. Pattern Anal. Mach. Intell. 22 (2000), 266- 280

[13] T. Preusser, M. Droske, C. S. Garbe, A. Telea, and M. Rumpf. 2007. A Phase Field Method For Joint Denoising, Edge Detection, And Motion Estimation. In Image Sequence Processing. Society for Industrial and Applied Mathematics, SIAM J. APPL. MATH.

[14] Y. Rathi, N. Vaswani, A. Tannenbaum, and A. Yezzi. 2005. Particle filtering for geometric active contours with application to tracking moving and deforming objects. In Proceedings of the 2005 IEEE Computer Society Conference on Computer Vision and Pattern Recognition (CVPR'05), IEEE Computer Society. 2(2005), 2-9.

[15] P. Puiseux. 2006. Analyse discrète : approximations polynomiales, 1-4.

[16] Gonzalez C.R. and R. 1992. Woods Digital Image Processing, Addison Wesley, 414-428.

[17] Jean-Michel JOLION. 1994. Multiresolution Analysis of Contrast in Digital Images. Laboratory of Computer systems Industrial Production and Signal Processing, 11(March 1994), 245-255.

[18] S. L. Keeling and W. Ring. 2005. Medical image registration and interpolation by optical flow with maximal rigidity, J. Math. Imaging Vision, 23 (2005), $47-65$

[19] P. Kornprobst, R. Deriche. 1999. and G. Aubert, Image sequence analysis via partial differential equations, J. Math. Imaging Vision, 11 (1999), 5-26.

[20] M. Lagzouli, Y. Elkettani. 2012. Traitement d'Image par Differences Finies et la Notion du Contour. Au colloque Modélisation et Simulation Numérique Méthode des Eléments finis et Ondelettes «MNOTSI-2012 » à l'école des ingénieurs -ENSA- université ibn Tofail KenitraMaroc.

[21] H.-H. Nagel and W. 1986. Enkelmann, An investigation of smoothness constraints for the estimation of displacement vector fields from image sequences, IEEE Trans. Pattern Anal. Mach. Intell., 8 (1986), 565-593.

[22] P. Nesi. 1993. Variational approach to optical flow estimation managing discontinuities, Image Vision Comput., 11 (1993), 419-439. 